\title{
Flexible and Shape-Reconfigurable Hydrogel Interlocking Adhesives for High Adhesion in Wet Environments Based on Anisotropic Swelling of Hydrogel Microstructures
}

Hyun-Ha Park, $^{\dagger}$ Minho Seong, $^{\dagger}$ Kahyun Sun $^{\dagger}$ Hangil Ko, $^{\dagger}$ Sang Moon Kim, ${ }^{\dagger}$ and Hoon Eui Jeong ${ }^{*}{ }^{\dagger} \odot$

${ }^{\dagger}$ Department of Mechanical Engineering, Ulsan National Institute of Science and Technology (UNIST), Ulsan 44919, Republic of Korea

${ }^{\ddagger}$ Department of Mechanical Engineering, Incheon National University, Incheon 22012, Republic of Korea

Supporting Information

ABSTRACT: This study presents wet-responsive, shape-reconfigurable, and flexible hydrogel adhesives that exhibit strong adhesion under wet environments based on reversible interlocking between reconfigurable microhook arrays. The experimental investigation on the swelling behavior and structural characterization of the hydrogel microstructures reveal that the microhook arrays undergo anisotropic swelling and shape transformation upon contact with water. The adhesion between the interlocked microhook arrays is greatly enhanced under wet conditions because of the hydrationtriggered shape reconfiguration of the hydrogel microstructures. Furthermore, wet adhesion monotonically increases with waterexposure time. A maximum adhesion force of $79.9 \mathrm{~N} \mathrm{~cm}^{-2}$ in the shear direction is obtained with the hydrogel microhook array after $20 \mathrm{~h}$ of swelling, which is $732.3 \%$ greater than that under dry conditions (i.e., $9.6 \mathrm{~N} \mathrm{~cm}^{-2}$ ). A simple theoretical model is developed to describe the measured adhesion forces. The results are in good agreement with the experimental data.
$S_{\text {the }}^{\text {the }}$ table adhesion between surfaces under wet conditions is highly desirable for many practical applications, particularly in the bioengineering and medical fields, where most surfaces are wet. $^{1-4}$ Demands for reversible wet adhesives are also increasing in nanotransfer printing for the fabrication of flexible electronic devices. ${ }^{5-7}$ Stable binding between functional layers (e.g., electrode, catalyst, and porous membrane) without delamination is also a prerequisite for stretchable electronic devices and energy devices operating under wet conditions. ${ }^{8-10}$ Wet adhesives have useful applications not only in the engineering field, but also in our daily lives. However, the stable adhesion of two components in wet environments is challenging because water molecules can easily penetrate the gaps between the surfaces, and the performance of adhesive materials deteriorates because of hydration-induced softening and dissolution. ${ }^{4,11,12}$ One representative effort to overcome such difficulties includes the development of a synthetic wet adhesive mimicking mussel foot proteins decorated with amino acid 3,4-dihydroxy-L-phenyl alanine (DOPA). ${ }^{13,14}$ However, DOPA-based adhesives face limitations, such as irreversible adhesion, performance degradation by wet air oxidation, and high cost. Other chemical-based wet adhesives, such as cyanoacrylate, also face limitations, including low adhesion strength, toxicity, requirements for specific functional groups, and hydration-induced dissolution. ${ }^{2}$ Furthermore, they are typically permanent adhesives, and therefore, have limitations for application as a reversible and reusable adhesive.

A miniaturized fastener utilizing interlocking between microor nanoscale structures could be an effective solution to ensure reversible adhesion in wet environments. Inspired by biological interlocking systems, ${ }^{15,16}$ various micro- and nanointerlocking devices have recently been developed using nanowires, carbon nanotubes, and polymeric micro- and nanostructures with high aspect ratios. ${ }^{17-19}$ These devices provide strong and reversible shear adhesion based on amplified van der Waals interactions between interlocked micro- or nanostructures. However, most of these devices have been tested and used only under dry conditions, and their interlocking mechanism under wet conditions has rarely been reported. Our preliminary experiments showed that the interlocking between high-aspect ratio micro- or nanopillar structures easily collapse under wet conditions because of capillary-induced lateral or bottom collapses. Therefore, reversible and strong adhesion and binding in wet or underwater environments are still elusive.

In this study, we present a flexible, wet-responsive, and reconfigurable hydrogel interlocking adhesive with microhook

Received: October 23, 2017

Accepted: November 13, 2017

Published: November 14, 2017 
a

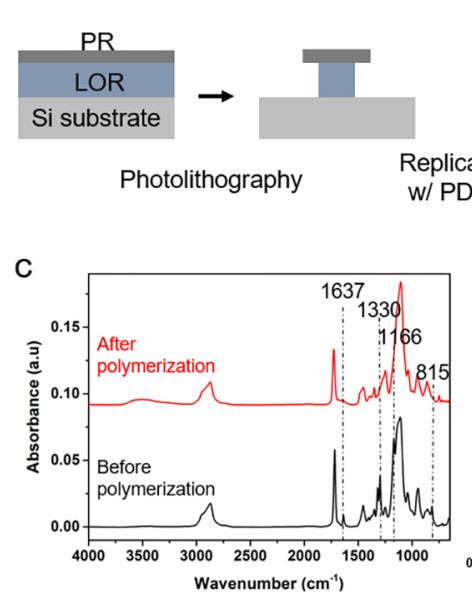

b
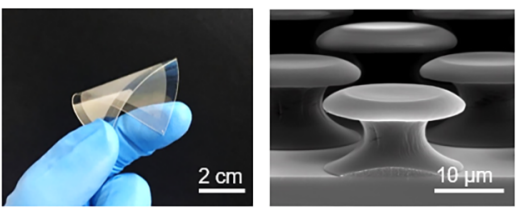

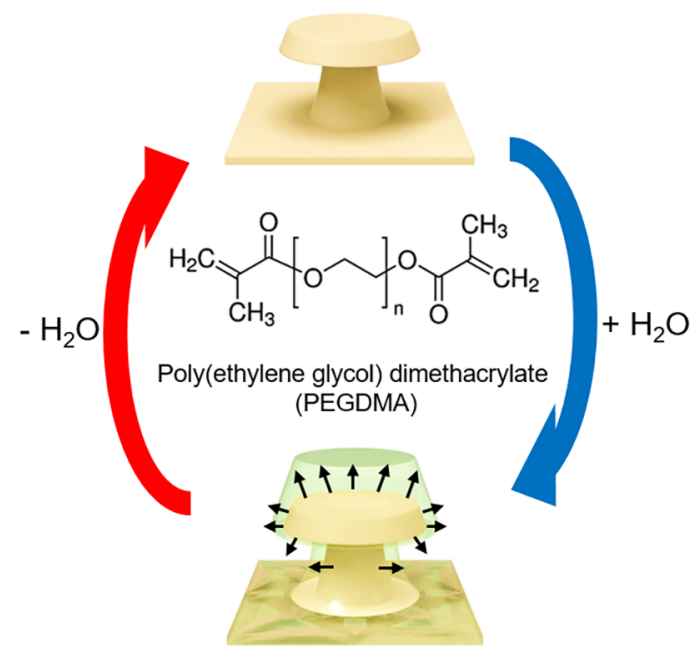

f
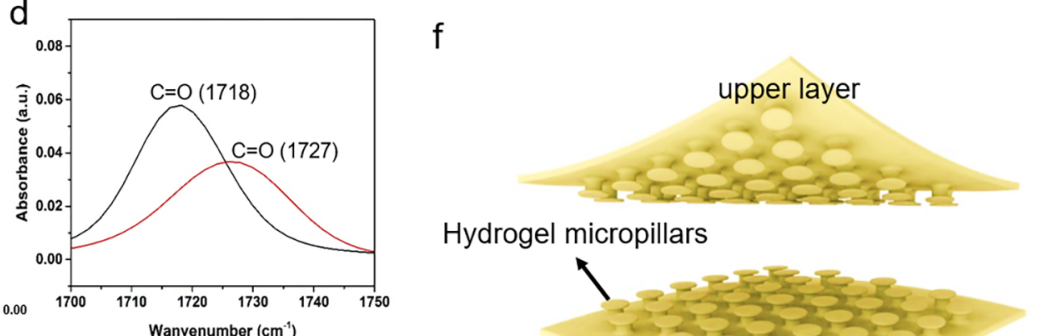

Hydrogel micropillars

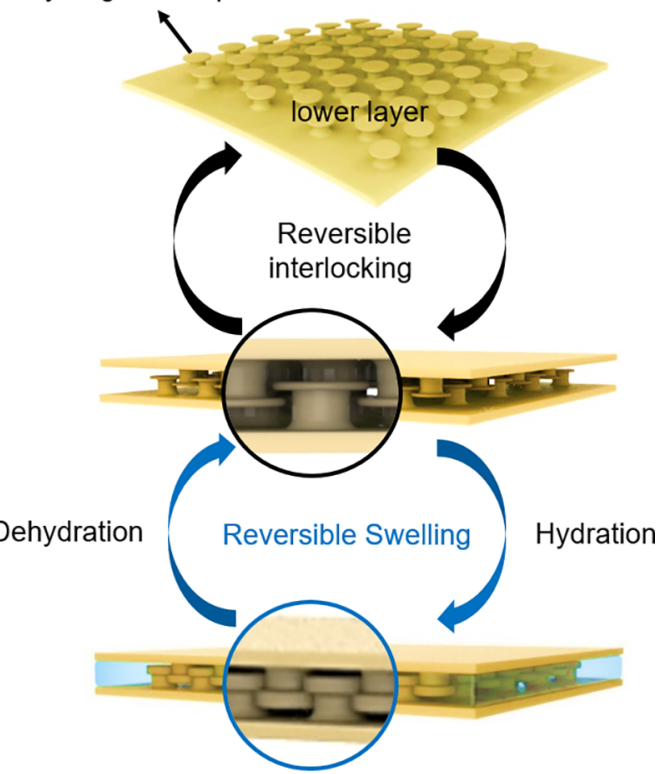

Figure 1. (a) Schematic of the fabrication of the wet-responsive and shape-reconfigurable PEGDMA interlocking adhesive with microhook arrays. (b) Photograph of the fabricated flexible interlocking adhesive (left) and a scanning electron microscopy (SEM) image of the PEGDMA microhook arrays of the adhesive. (c) ATR-FTIR spectra of the PEGDMA monomers and photopolymerized samples in the wavenumber range of 650-4000 $\mathrm{cm}^{-1}$. (d) Detailed view of the ATR-FTIR spectra of the PEGDMA monomers and the photopolymerized samples in the wavenumber range of $1700-1750 \mathrm{~cm}^{-1}$. (e) Conceptual illustration of the reversible swelling and deswelling process of the PEGDMA microhooks. (f) Conceptual illustration of the reversible interlocking of the PEGDMA microhook arrays via the hydration-induced shape reconfiguration of the array for high adhesion under wet conditions.

arrays that exhibits a strong and reversible interlocking adhesion in wet or underwater conditions based on the hydration-triggered swelling behaviors of hydrogel polymers. A microstructural interpenetration occurs between the mating arrays when two identical hydrogel microhook arrays are brought into contact under dry conditions, resulting in relatively weak adhesion. Upon exposure to water, a significant volume expansion and a corresponding shape transformation of the hydrogel microhooks occur because of the anisotropic swelling of the hydrogel, ${ }^{20}$ which leads to an increase in their structural bending strength, contact surface, and friction with the neighboring microstructures. Consequently, enhanced and reversible wet adhesion can be achieved in underwater conditions with simple hydrogel microstructures alone without the need for extensive surface modification with chemicals. Furthermore, the wet adhesion strength increases with the increasing water-exposure time, which is a counterintuitive behavior with conventional chemical adhesives that significantly lose adhesion capability when exposed to moisture or water.
Theoretical models are proposed to evaluate the interlocking adhesion strength based on a simple force balance analysis.

We fabricate a microscale hook array by replica molding with polyethylene glycol dimethacrylate (PEGDMA) to develop a fastener-type adhesive that exerts strong adhesion under wet environments (Figure 1a, see Supporting Information and Figure S1 for the fabrication details). PEGDMA is a hydrogel capable of absorbing large quantities of water or physiological solutions, and can be cured by exposure to ultraviolet (UV) light, which enables a rapid and scalable fabrication of complicated micro- or nanoscale structures. ${ }^{21,22}$ Aside from the PEGDMA hydrogel, other swellable hydrogels in response to external stimuli (e.g., poly(2-hydroxyethyl methacrylate), poly ( $N$-isopropylacrylamide), and poly acrylic acid) could also be utilized for shape reconfigurable smart interlocking adhesive systems. ${ }^{23,24}$ Figure $1 \mathrm{~b}$ shows the fabricated PEGDMA adhesive with microhook arrays. The PEGDMA adhesive was in the form of a thin flexible film and can be integrated into various devices. The microhooks of the adhesive were designed to exhibit a unique structural configuration with protruding heads 
and played an important role in securing an effective interlocking between two mating arrays. Figure $1 \mathrm{c}, \mathrm{d}$ shows the attenuated total reflectance-Fourier transform infrared (ATR-FTIR) spectra of the PEGDMA monomers and the photopolymerized hydrogel samples. A decrease in the alkene stretching peak at $1637 \mathrm{~cm}^{-1}$ was observed, indicating that most of the $\mathrm{C}=\mathrm{C}$ bonds in the monomers reacted under our fabrication conditions. A shift of the peak at $1718-1727 \mathrm{~cm}^{-1}$ was also observed for the polymerized hydrogel, implying a change in the chemical environment in the vicinity of the $\mathrm{C}=$ $\mathrm{O}$ bond because of the conversion of $\mathrm{C}=\mathrm{C}$ to $\mathrm{C}-\mathrm{C}{ }^{25}$ Water was entrapped in the three-dimensional (3D) polymer networks when the photopolymerized PEGDMA was exposed to water, thereby resulting in a significant volume expansion. As a result, the shapes of the PEGDMA microstructures became larger and expanded with a notable increase in the structural diameter and height because of the swelling with water (Figure 1e). This water-responsive shape change was reversible, and the microstructure can recover its original shape and size upon water removal by drying (deswelling). A hydration-triggered shape transformation can be observed in pine cones and plants, such as Acanthoideae. ${ }^{26-28}$ Such a humidity-triggered shape reconfiguration was exploited to realize strong adhesion under wet conditions in our hydrogel fastener-type adhesives. When the two PEGDMA films with reconfigurable microhook arrays were brought into contact in the dry state, the microhooks easily interpenetrated each other at a low preload. A significant volume expansion occurred when the interlocked sample was hydrated in response to humidity or water, enabling strong wet adhesion through enhanced interlocking and tight contact between the neighboring microhooks (Figure 1f).

The swelling behavior of the PEGDMA film was quantitatively evaluated as a function of time to investigate the shape transformation behavior of the fabricated PEGDMA film in response to humidity by submerging the hydrogel film in water. As shown in Figure 2a, the swelling ratio (\%), which was defined as $\frac{W_{\mathrm{s}}-W_{\mathrm{d}}}{W_{\mathrm{d}}} \times 100\left(W_{\mathrm{s}}\right.$ and $W_{\mathrm{d}}$ are the weights of the hydrogel in the swollen and dried states, respectively), gradually increased with time. The swelling ratio was $28.0 \% \pm 2.8 \%$ after $20 \mathrm{~h}$ of swelling. Consequently, the macroscopic size of the PEGDMA sample notably increased with an increase in the water-exposure time (Figure $2 \mathrm{~b}$ ). The tip diameter of the individual microhooks also increased with the water-submerging time. The initial tip diameter of $20.3 \mu \mathrm{m}$ increased to 21.8 , 23.0, 24.3, and $25.1 \mu \mathrm{m}$ after $0.5,1,4$, and $20 \mathrm{~h}$ swelling, respectively. The increase rate of the tip diameter was high at the beginning of the swelling, then gradually reduced with time. Figure $2 \mathrm{c}, \mathrm{d}$ depicts the optical microscope images of the microhooks before and after hydration. The tip diameter of the microstructures noticeably increased from 20.3 to $25.1 \mu \mathrm{m}$ (23.6\% increase), while the space between them was reduced. The $3 \mathrm{D}$ reconstruction images of the microhook structures using confocal microscopy indicated a dramatic hydrationtriggered shape transformation (Figure 2e,f).

The increase in the size of the microhooks was more remarkable in the structural height direction rather than in the lateral direction, because when the hydrogel film was formed on a rigid substrate, the hydrogel preferentially swelled in the direction normal to the surface due to the mechanical constraint provided by the substrate. ${ }^{29}$ The initial heights of the microstructures $(13.0 \mu \mathrm{m})$ were increased to $17.8 \mu \mathrm{m}$ after $20 \mathrm{~h}$ of swelling, indicating an enhancement of $36.9 \%$.
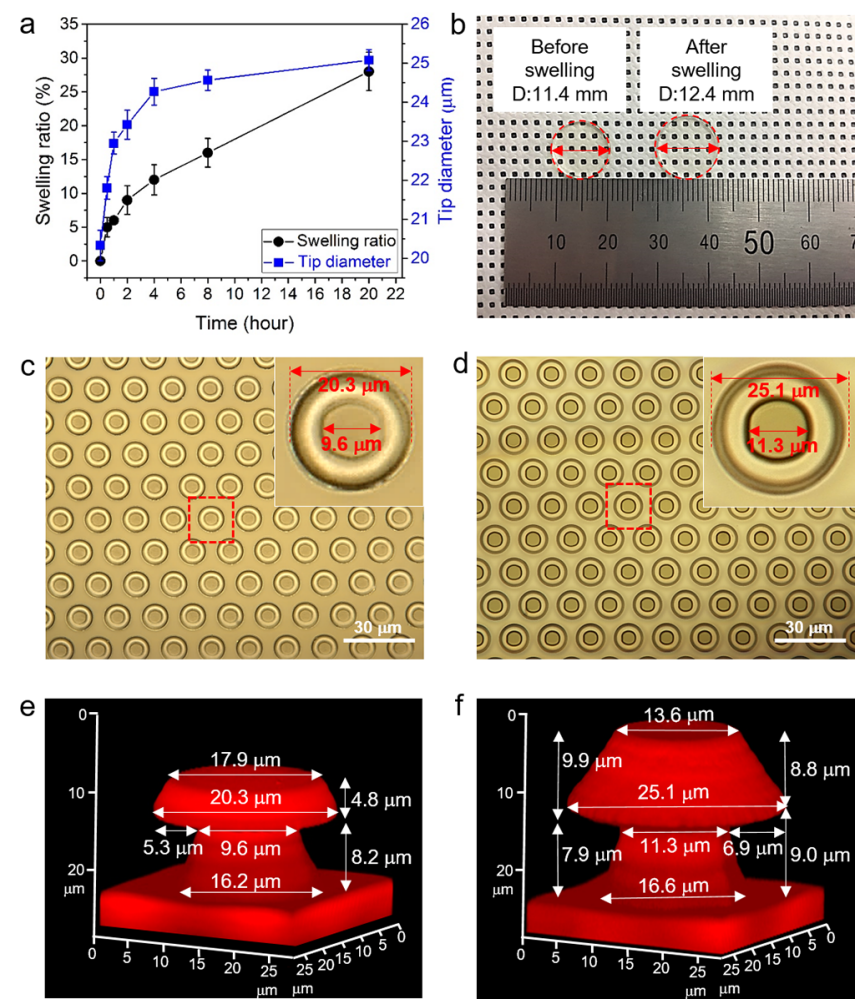

Figure 2. (a) Swelling ratio of the PEGDMA adhesive and tip diameter of the microhooks as functions of the water-exposure time. (b) Photograph showing the macroscopic changes in the size of the PEGDMA sample upon swelling with water. Optical microscope images of the microhooks (c) before and (d) after swelling. 3D reconstruction images of the microhook structures (e) before and (f) after swelling produced using confocal microscopy.

Interestingly, the tip thickness more dramatically increased by $106.3 \%$ from 4.8 to $9.9 \mu \mathrm{m}$ after $20 \mathrm{~h}$ of swelling because the tip of the microhook underwent swelling from both the top and bottom sides of the tip, whereas osmotic swelling at the microhook's stem/substrate interface was restricted.

Note that while the overall tip diameters and thicknesses increased, the diameter of the top surface of the tip decreased from 17.9 to $13.6 \mu \mathrm{m}$ after swelling, which seemed to be caused by the large compressive stress at the top surface of the tip. According to a previous study, structures with a low aspect ratio (structural height over diameter) constrained by a substrate undergo a large compressive stress at the top surface, ${ }^{30,31}$ which could result in a decrease in diameter of the top surface. The degree of swelling of the hydrogel can be modulated by using hydrogels with different molecular weights. Typically, hydrogels with larger molecular weights exhibit larger volume changes as they can absorb more water molecules. ${ }^{32}$ However, it is wellknown that hydrogels with higher molecular weights have lower mechanical strength after curing, ${ }^{33}$ which would lower the allowable interlocking strength of the hydrogel adhesives. In this study, we used PEGDMA with a molecular weight (MW) of 550 to achieve a balance between the degree of swelling and the mechanical strength.

To examine the interlocking behavior of the PEGDMA microhooks under wet conditions, the interlocking shear and normal strength of the PEGDMA adhesives were measured with a custom-built adhesion force measurement system (see Supporting Information and Figure S2 for details). ${ }^{34}$ To investigate the effects of geometry on the interlocking behavior, 
various microhook arrays with different pitches $(25,30,35$, and $40 \mu \mathrm{m}$ ) were interlocked by bringing two identical PEGDMA films into contact with each other at a preload of $10 \mathrm{~N} \mathrm{~cm}^{-2}$ (Figure 3a). Figure 3b,c shows the fluorescent and optical
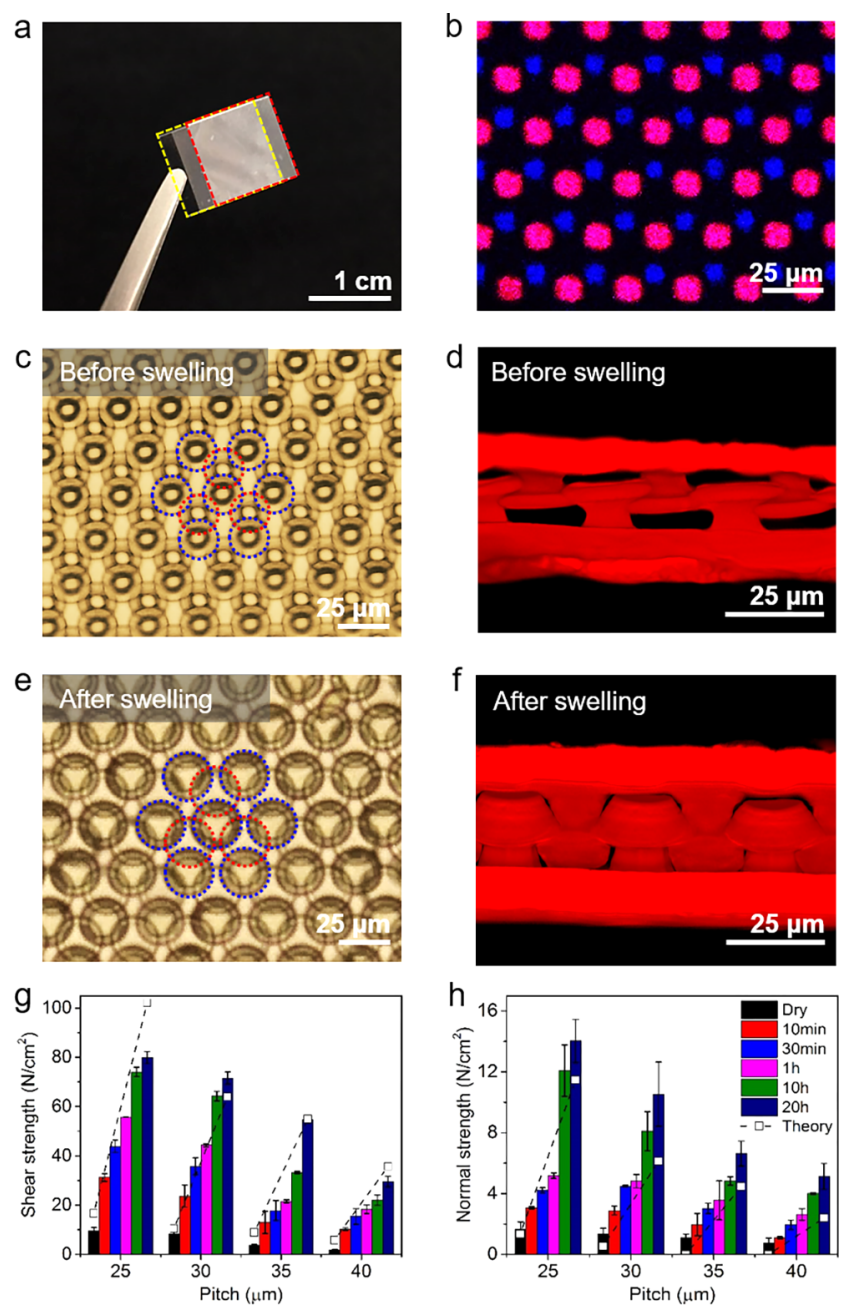

Figure 3. (a) Photograph of the interlocked PEGDMA adhesives with microhook arrays. (b) Fluorescent, (c) optical, and (d) 3D confocal microscope images of the interlocked PEGDMA microhook arrays with a $25 \mu \mathrm{m}$ pitch in the dry state. (e) Optical and (f) 3D confocal microscope images of the interlocked PEGDMA microhook arrays with a $25 \mu \mathrm{m}$ pitch in the swollen state. (g) Shear and (h) normal interlocking adhesion strengths of the interlocked microhook arrays with four different pitches $(25,30,35$, and $40 \mu \mathrm{m})$ as functions of the swelling time. Dashed lines in $(\mathrm{g})$ and $(\mathrm{h})$ indicate the theoretical predictions of the adhesion strengths of the interlocked arrays.

microscopic images of the interlocked arrays with a pitch of 25 $\mu \mathrm{m}$. A 3D reconstruction image of the interlocked array using confocal microscopy are also presented in Figure 3d. As shown, the microhooks successfully interpenetrated each other and assembled into a single interlocked form with a self-alignment capability in the dry state by the application of external pressures. The tips of the microhook arrays slightly overlapped with the tips of the counter array in the dry state. When the interlocked adhesives were exposed to water, hydration-induced shape reconfiguration occurred, resulting in volume expansion and a subsequent increase in the overlapping area of the microhook structures (Figure 3e,f). Figure 3g,h demonstrates the relationship between the measured interlocking strength of the PEGDMA adhesives with different pitches and swelling time. As shown, the hydrogel adhesives exhibited a relatively low shear $\left(<10 \mathrm{~N} \mathrm{~cm}^{-2}\right)$ and normal $\left(<2 \mathrm{~N} \mathrm{~cm}^{-2}\right)$ adhesion in dry conditions. However, when the interlocked hydrogel adhesives were exposed to water, the adhesion strength increased significantly with an increase in the swelling time due to swelling-induced shape changes in the microhook arrays. For example, the shear strength of the microhook array with a $25 \mu \mathrm{m}$ pitch increased by $210.0 \%$ from $10 \mathrm{~N} \mathrm{~cm}^{-2}$ under dry conditions to $31 \mathrm{~N} \mathrm{~cm}^{-2}$, after just $10 \mathrm{~min}$ of exposure to water. The increase in the adhesion strength of the hydrogels with an increase in the water-exposure time is counterintuitive to the behavior of conventional chemical adhesives, whose adhesion strength decreases with an increase in the water- or moistureexposure time. The shear strength of the samples with a pitch of $25 \mu \mathrm{m}$ increased monotonically to $44,56,74$, and $80 \mathrm{~N} \mathrm{~cm}^{-2}$ after $0.5,1,10$, and $20 \mathrm{~h}$ of hydration, respectively (Figure $3 \mathrm{~g}$ ). In addition to the remarkably high shear strength, the PEGDMA adhesives also exhibited a high wet adhesion strength in the normal direction (maximum $14 \mathrm{~N} \mathrm{~cm}^{-2}$, Figure $3 \mathrm{~h})$; on the other hand, previously reported micro- or nanointerlocking devices showed nearly zero or low normal strength $\left(\sim 6-8 \mathrm{~N} \mathrm{~cm}^{-2}\right) .{ }^{35}$ The shear and normal adhesion strengths were saturated after $20 \mathrm{~h}$ swelling (Figure S3). PEGDMA adhesives with smaller pitches exhibited higher shear and normal strengths, due to the fact that the arrays with smaller pitches have larger overlapping areas and tighter contact with the neighboring structures as well as higher structural densities. Repeatability and reversibility of the PEGDMA adhesives were also investigated by measuring adhesion strength of the adhesives during repeated cycles of swelling and deswelling (Figure S4). The PEGDMA adhesive showed a reversible and repeatable interlocking adhesion performance without a notable adhesion degradation and a structural failure during the durability tests (Figures S4 and S5).

To further understand the interlocking behavior of the fastener, we derived a theory based on the force balance between the microhook arrays (see Supporting Information for details). Using the force equilibrium of a single paired interlocked microhook array, the normal interlocking adhesion force $\left(F_{\text {normal }}\right)$ can be given as

$$
F_{\text {normal }}=n F_{\text {ext }}=n\left(F_{\mathrm{b}, \mathrm{t}}+\frac{1}{3} F_{\mathrm{ad}}+f_{1}+f_{2}\right)
$$

where $n$ is the number of microhooks per unit area, $F_{\text {ext }}$ is the external force acting on the single microhook, $F_{b, t}$ is the bending force acting on the tip, $F_{\text {ad }}$ is the adhesion force between the tip and the bottom substrates, and $f_{1}$ and $f_{2}$ are the frictional forces between the side walls of the stem and the tips of the interlocked microhooks, respectively (see Figures S6a and S6b). The shear adhesion force $\left(F_{\text {shear }}\right)$ can be written as

$$
F_{\text {shear }}=n F_{\text {ext }} \cos \left(\theta_{\text {ext }}\right)=n\left[F_{x} \cos \left(\frac{\pi}{2}-\theta_{\mathrm{m}}\right)+F_{y} \cos \left(\theta_{\mathrm{m}}\right)\right]
$$

where $\theta_{\text {ext }}$ is the angle of external force from a horizontal surface and $\theta_{\mathrm{m}}$ is the tilting angle of the microhook. $F_{x}$ and $F_{y}$ are the $x$ - and $y$-components of the external force (Figure $S 6 c, d)$. Our theoretical analysis shows that the volume expansion of the individual microhooks and subsequent changes in the microstructural geometries results in the enhanced interlocking strength of the PEGDMA adhesive. 

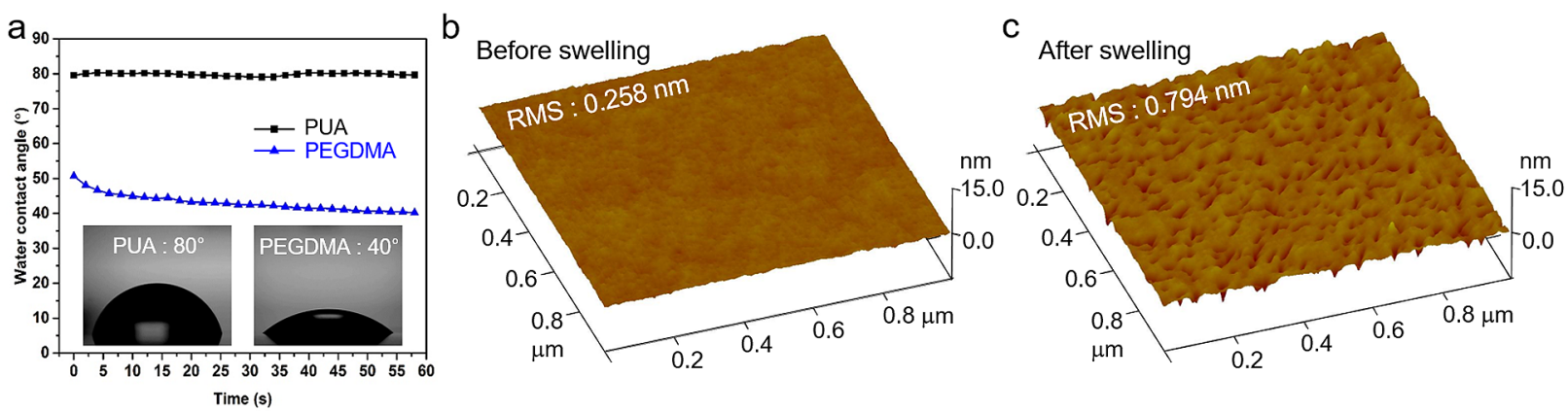

Figure 4. (a) Variation in the contact angles of water droplets on PUA and PEGDMA films with time. RMS roughness values of the PEGDMA films (b) before and (c) after swelling for $20 \mathrm{~h}$, as measured by AFM.

The theoretical predictions well agreed with the experimental results, as shown in Figure S6e,f, demonstrating the validity of our analytical models.

To better understand the interlocking mechanism of the PEGDMA adhesive, the surface wetting, surface roughness, and elastic moduli of the PEGDMA samples were analyzed. Figure 4a shows the dynamic contact angles (CAs) of deionized (DI) water on a planar PEGDMA film and a polyurethane acrylate (PUA) film. PUA is UV-curable resin, which does not exhibit water swelling. ${ }^{36,37}$ As shown in Figure 4a, the CA of DI water on the PUA film remains at $80^{\circ}$ irrespective of the passage of time. In contrast, the CA of DI water on the PEGDMA film decreased monotonically from an initial CA of $51^{\circ}$. This indicates that PEGDMA swells continuously when in contact with water. Figure $4 b, c$ depicts the surface roughness of the PEGDMA samples, as measured by atomic force microscopy (AFM), before and after hydration with water for $20 \mathrm{~h}$. As shown, the root-mean-square (RMS) roughness of the film increased slightly from $0.26 \mathrm{~nm}$ for the dry sample to $0.79 \mathrm{~nm}$ for the $20 \mathrm{~h}$-swollen sample. According to additional adhesion measurement experiments, the increase in RMS from 0.26 to $0.79 \mathrm{~nm}$ showed negligible effects to the adhesion strengths (Figure S10). The elastic moduli of the PEGDMA samples in dry and swollen states are shown in Table 1. Young's moduli of

Table 1. Young's Moduli and Elongations at Break of the PEGDMA Films in the Dry and Swollen States in Water after $20 \mathrm{~h}$ of Exposure

\begin{tabular}{lcc} 
& dry state & swollen state (in water) \\
\hline Young's modulus $(\mathrm{MPa})$ & $61.7 \pm 6.5$ & $60.3 \pm 6.6$ \\
elongation at break $(\%)$ & $10.2 \pm 0.7$ & $5.1 \pm 0.6$ \\
\hline
\end{tabular}

the PEGDMA films were almost the same before $(61.7 \mathrm{MPa})$ and after $(60.3-62.3 \mathrm{MPa}) 20 \mathrm{~h}$ of swelling in water. Elongation at break was decreased after swelling. These results indicate that the surface roughness or mechanical strength of the material is not directly related to the enhanced interlocking strength of the PEGDMA adhesive.

In summary, we developed a wet-responsive and reversible fastener-type adhesive based on the hydration-induced anisotropic swelling behaviors of hydrogel arrays. When two hydrogel microhook arrays were brought into contact with each other under dry conditions, a relatively low adhesion was observed. However, when the interlocked arrays were exposed to water, a notable volume expansion and a corresponding shape transformation of the hydrogel microhooks occurred by the swelling of the hydrogel, resulting in significantly increased wet adhesion both in the shear $\left(80 \mathrm{~N} \mathrm{~cm}^{-2}\right)$ and normal $(14 \mathrm{~N}$ $\mathrm{cm}^{-2}$ ) directions. In contrast to other wet binding systems, the current interlocking mechanism does not involve any complicated surface treatment or chemical moieties, thus allowing for a simple yet efficient route to strong and reversible wet adhesion in a cost-effective manner. This wet-responsive and reversible hydrogel interlocking adhesive can serve as a robust and versatile wet adhesive for a broad range of applications which require stable and strong adhesion under diverse wet conditions.

\section{ASSOCIATED CONTENT}

\section{S Supporting Information}

The Supporting Information is available free of charge on the ACS Publications website at DOI: 10.1021/acsmacrolett.7b00829.

Experimental details (PDF).

\section{AUTHOR INFORMATION}

\section{Corresponding Author}

*E-mail: hoonejeong@unist.ac.kr. Tel.: +82-52-217-2339.

ORCID

Hoon Eui Jeong: 0000-0002-1413-3774

Notes

The authors declare no competing financial interest.

\section{ACKNOWLEDGMENTS}

This work was supported by the National Research Foundation of Korea (NRF) Grant funded by the Ministry of Science, ICT \& Future Planning for convergent research in Nature-Inspired Innovative Technology Development Program (NRF2017M3C1B7014238) and the Midcareer Researchers Supporting Program (2016R1A2B2014044).

\section{REFERENCES}

(1) Oh, D. X.; Kim, S.; Lee, D.; Hwang, D. S. Tunicate-mimetic nanofibrous hydrogel adhesive with improved wet adhesion. Acta Biomater. 2015, 20, 104-112.

(2) Oh, D. X.; Shin, S.; Yoo, H. Y.; Lim, C.; Hwang, D. S. Surface forces apparatus and its applications for nanomechanics of underwater adhesives. Korean J. Chem. Eng. 2014, 31, 1306-1315.

(3) Mahdavi, A.; Ferreira, L.; Sundback, C.; Nichol, J. W.; Chan, E. P.; Carter, D. J. D.; Bettinger, C. J.; Patanavanich, S.; Chignozha, L.; Ben-Joseph, E.; Galakatos, A.; Pryor, H.; Pomerantseva, I.; Masiakos, P. T.; Faquin, W.; Zumbuehl, A.; Hong, S.; Borenstein, J.; Vacanti, J.; Langer, R.; Karp, J. M. A biodegradable and biocompatible geckoinspired tissue adhesive. Proc. Natl. Acad. Sci. U. S. A. 2008, 105, 2307-2312. 
(4) Thai, T. H. T.; Lee, J. S.; Lee, Y.; Park, H. B.; Park, K. M.; Park, K. D. Supramolecular cyclodextrin supplements to improve the tissue adhesion strength of gelatin bioglues. ACS Macro Lett. 2017, 6, 83-88.

(5) Lee, H.; Um, D. S.; Lee, Y.; Lim, S.; Kim, H. J.; Ko, H. Octopusinspired smart adhesive pads for transfer printing of semiconducting nanomembranes. Adv. Mater. 2016, 28, 7457-7465.

(6) Lee, C. H.; Kim, D. R.; Zheng, X. L. Fabrication of nanowire electronics on nonconventional substrates by water-assisted transfer printing method. Nano Lett. 2011, 11, 3435-3439.

(7) Lee, B. H.; Cho, Y. H.; Lee, H.; Lee, K. D.; Kim, S. H.; Sung, M. $\mathrm{M}$. High-resolution patterning of aluminum thin films with a watermediated transfer process. Adv. Mater. 2007, 19, 1714-1718.

(8) Liu, Z. Y.; Wang, X. T.; Qi, D. P.; Xu, C.; Yu, J. C.; Liu, Y. Q.; Jiang, Y.; Liedberg, B.; Chen, X. D. High-adhesion stretchable electrodes based on nanopile interlocking. Adv. Mater. 2017, 29, 1603382.

(9) Yuk, S.; Choo, M. J.; Lee, D.; Guim, H.; Kim, T. H.; Lee, D. G.; Choi, S.; Lee, D. H.; Doo, G.; Hong, Y. T.; Kim, H. T. Threedimensional interlocking interface: mechanical nanofastener for high interfacial robustness of polymer electrolyte membrane fuel cells. Adv. Mater. 2017, 29, 1603056.

(10) Oh, K. H.; Kang, H. S.; Choo, M. J.; Jang, D. H.; Lee, D.; Lee, D. G.; Kim, T. H.; Hong, Y. T.; Park, J. K.; Kim, H. T. Interlocking membrane/catalyst layer interface for high mechanical robustness of hydrocarbon-membrane-based polymer electrolyte membrane fuel cells. Adv. Mater. 2015, 27, 2974-2980.

(11) Yamate, T.; Kumazawa, K.; Suzuki, H.; Akazome, M. CH/pi interactions for macroscopic interfacial adhesion design. ACS Macro Lett. 2016, 5, 858-861.

(12) Deng, G. H.; Ma, Q.; Yu, H. X.; Zhang, Y. F.; Yan, Z. C.; Liu, F. Y.; Liu, C. Y.; Jiang, H. F.; Chen, Y. M. Macroscopic organohydrogel hybrid from rapid adhesion between dynamic covalent hydrogel and organogel. ACS Macro Lett. 2015, 4, 467-471.

(13) Lee, B. P.; Messersmith, P. B.; Israelachvili, J. N.; Waite, J. H. Mussel-inspired adhesives and coatings. Annu. Rev. Mater. Res. 2011, 41, 99-132.

(14) Brubaker, C. E.; Messersmith, P. B. The present and future of biologically inspired adhesive interfaces and materials. Langmuir 2012, 28, 2200-2205.

(15) Pang, C.; Kim, T. I.; Bae, W. G.; Kang, D.; Kim, S. M.; Suh, K. Y. Bioinspired reversible interlocker using regularly arrayed high aspect-ratio polymer fibers. Adv. Mater. 2012, 24, 475-479.

(16) Gorb, S. N. Biological attachment devices: exploring nature's diversity for biomimetics. Philos. Trans. R. Soc., A 2008, 366, 15571574.

(17) Chen, C. M.; Chiang, C. L.; Lai, C. L.; Xie, T.; Yang, S. Buckling-based strong dry adhesives via interlocking. Adv. Funct. Mater. 2013, 23, 3813-3823.

(18) Lee, C.; Kim, S. M.; Kim, Y. J.; Choi, Y. W.; Suh, K. Y.; Pang, C.; Choi, M. Robust microzip fastener: repeatable interlocking using polymeric rectangular parallelepiped arrays. ACS Appl. Mater. Interfaces 2015, 7, 2561-2568.

(19) Ko, H.; Zhang, Z. X.; Chueh, Y. L.; Ho, J. C.; Lee, J.; Fearing, R. S.; Javey, A. Wet and dry adhesion properties of self-selective nanowire connectors. Adv. Funct. Mater. 2009, 19, 3098-3102.

(20) Guvendiren, M.; Yang, S.; Burdick, J. A. Swelling-induced surface patterns in hydrogels with gradient crosslinking density. Adv. Funct. Mater. 2009, 19, 3038-3045.

(21) Suh, K. Y.; Langer, R.; Lahann, J. A novel photodefinable reactive polymer coating and its use for microfabrication of hydrogel elements. Adv. Mater. 2004, 16, 1401-1405.

(22) Suh, K. Y.; Seong, J.; Khademhosseini, A.; Laibinis, P. E.; Langer, R. A simple soft lithographic route to fabrication of poly(ethylene glycol) microstructures for protein and cell patterning. Biomaterials 2004, 25, 557-563.

(23) Tomic, S. L.; Micic, M. M.; Filipovic, J. M.; Suljovrujic, E. H. Swelling and drug release behavior of poly(2-hydroxyethyl methacrylate/itaconic acid) copolymeric hydrogels obtained by gamma irradiation. Radiat. Phys. Chem. 2007, 76, 801-810.
(24) Elliott, J. E.; Macdonald, M.; Nie, J.; Bowman, C. N. Structure and swelling of poly(acrylic acid) hydrogels: effect of $\mathrm{pH}$, ionic strength, and dilution on the crosslinked polymer structure. Polymer 2004, 45, 1503-1510.

(25) Hwang, J. W.; Noh, S. M.; Kim, B.; Jung, H. W. Gelation and crosslinking characteristics of photopolymerized poly(ethylene glycol) hydrogels. J. Appl. Polym. Sci. 2015, 132, 41939.

(26) Armon, S.; Efrati, E.; Kupferman, R.; Sharon, E. Geometry and mechanics in the opening of chiral seed pods. Science 2011, 333, $1726-1730$.

(27) Studart, A. R.; Erb, R. M. Bioinspired materials that self-shape through programmed microstructures. Soft Matter 2014, 10, 12841294.

(28) Rafsanjani, A.; Brule, V.; Western, T. L.; Pasini, D. Hydroresponsive curling of the resurrection plant selaginella lepidophylla. Sci. Rep. 2015, 5, 8064

(29) Guvendiren, M.; Burdick, J. A.; Yang, S. Kinetic study of swelling-induced surface pattern formation and ordering in hydrogel films with depth-wise crosslinking gradient. Soft Matter 2010, 6, 20442049.

(30) Kang, M. K.; Huang, R. A Variational approach and finite element implementation for swelling of polymeric hydrogels under geometric constraints. J. Appl. Mech. 2010, 77, 061004.

(31) Trujillo, V.; Kim, J.; Hayward, R. C. Creasing instability of surface-attached hydrogels. Soft Matter 2008, 4, 564-569.

(32) Park, S.; Lee, Y.; Kim, D. N.; Park, S.; Jang, E.; Koh, W. G. Entrapment of enzyme-linked magnetic nanoparticles within poly(ethylene glycol) hydrogel microparticles prepared by photopatterning. React. Funct. Polym. 2009, 69, 293-299.

(33) Temenoff, J. S.; Athanasiou, K. A.; LeBaron, R. G.; Mikos, A. G. Effect of poly(ethylene glycol) molecular weight on tensile and swelling properties of oligo(poly(ethylene glycol) fumarate) hydrogels for cartilage tissue engineering. J. Biomed. Mater. Res. 2002, 59, 429437.

(34) Yi, H.; Kang, M.; Kwak, M. K.; Jeong, H. E. Simple and reliable fabrication of bioinspired mushroom-shaped micropillars with precisely controlled tip geometries. ACS Appl. Mater. Interfaces 2016, $8,22671-22678$.

(35) Zhao, H. W.; Yue, Y. H.; Guo, L.; Wu, J. T.; Zhang, Y. W.; Li, X. D.; Mao, S. C.; Han, X. D. Cloning nacre's 3D interlocking skeleton in engineering composites to achieve exceptional mechanical properties. Adv. Mater. 2016, 28, 5099-5105.

(36) Kwak, R.; Park, H.-H.; Ko, H.; Seong, M.; Kwak, M. K.; Jeong, H. E. Partially cured photopolymer with gradient bingham plastic behaviors as a versatile deformable material. ACS Macro Lett. 2017, 6, $561-565$.

(37) Yi, H.; Hwang, I.; Lee, J. H.; Lee, D.; Lim, H.; Tahk, D.; Sung, M.; Bae, W. G.; Choi, S. J.; Kwak, M. K.; Jeong, H. E. Continuous and scalable fabrication of bioinspired dry adhesives via a roll-to-roll process with modulated ultraviolet-curable resin. ACS Appl. Mater. Interfaces 2014, 6, 14590-14599. 